\title{
Resolution of Born Scattering in Curve Geometries: Aspect-Limited Observations and Excitations
}

\author{
Ehsan Akbari Sekehravani $\left(D\right.$, Giovanni Leone * ${ }^{*}$ and Rocco Pierri $(\mathbb{D}$ \\ Department of Engineering, University of Campania “Luigi Vanvitelli", I-81031 Aversa, Italy; \\ ehsan.akbarisekehravani@unicampania.it (E.A.S.); rocco.pierri@unicampania.it (R.P.) \\ * Correspondence: Giovanni.leone@unicampania.it
}

check for updates

Citation: Sekehravani, E.A.; Leone, G.; Pierri, R. Resolution of Born Scattering in Curve Geometries: Aspect-Limited Observations and Excitations. Electronics 2021, 10, 3089. https://doi.org/10.3390/ electronics10243089

Academic Editors: Gian

Luigi Gragnani and

Alessandro Fedeli

Received: 4 November 2021

Accepted: 9 December 2021

Published: 12 December 2021

Publisher's Note: MDPI stays neutral with regard to jurisdictional claims in published maps and institutional affiliations.

Copyright: (C) 2021 by the authors. Licensee MDPI, Basel, Switzerland. This article is an open access article distributed under the terms and conditions of the Creative Commons Attribution (CC BY) license (https:/ / creativecommons.org/licenses/by/ $4.0 /)$.

\begin{abstract}
In inverse scattering problems, the most accurate possible imaging results require plane waves impinging from all directions and scattered fields observed in all observation directions around the object. Since this full information is infrequently available in actual applications, this paper is concerned with the mathematical analysis and numerical simulations to estimate the achievable resolution in object reconstruction from the knowledge of the scattered far-field when limited data are available at a single frequency. The investigation focuses on evaluating the Number of Degrees of Freedom (NDF) and the Point Spread Function (PSF), which accounts for reconstructing a point-like unknown and depends on the NDF. The discussion concerns objects belonging to curve geometries, in this case, circumference and square scatterers. In addition, since the exact evaluation of the PSF can only be accomplished numerically, an approximated closed-form evaluation is introduced and compared with the exact one. The approximation accuracy of the PSF is verified by numerical results, at least within its main lobe region, which is the most critical as far as the resolution discussion is concerned. The main result of the analysis is the space variance of the PSF for the considered geometries, showing that the resolution is different over the investigation domain. Finally, two numerical applications of the PSF concept are shown, and their relevance in the presence of noisy data is outlined.
\end{abstract}

Keywords: linear inverse scattering problem; point spread function; resolution; aspect-limited excitations; aspect-limited observations

\section{Introduction}

The inverse scattering problem is concerned with reconstructing some geometric and/or physical properties of an unknown object from the scattered field data under the illumination of known incident plane waves and to recover the permittivity, the permeability, and the shape of the object. Generally speaking, it is a nonlinear problem requiring careful formulation within appropriate solution spaces and leading to solution algorithms that have not been shown fully reliable. Therefore much attention is also devoted to linearized scattering models, which allow to provide full mathematical discussion and have many applications, such as radar and medical imaging. Hereafter we consider the prototype inverse scattering problem when plane waves excite the scattered field observed in the far zone for the 2D geometry, invariant along one axis.

Depending on the amount of information that can be collected, the inverse scattering problems can be categorized as full, or aspect limited, data problems [1-4]. In order to recover at best the characteristic of an object, it is required that the incident plane waves illuminate the object over from all angles, and then the scattered field is observed at all angles. Difficulties with this kind of problem immediately arise since this complete information is seldom available. Then, for the limited aspect case, incident plane waves impinge from a limited range of directions, and the scattered field pattern is observed only over a limited range of angles. Thus this problem is significant and challenging because, 
in practice, it is impossible to excite the incident plane wave and to measure the scattered field in all directions around an object. When full-space measurements are not available, the ill-posedness and nonlinearity of the inverse problem become more severe.

In linear inverse scattering imaging, the achievable resolution can be determined by the imaging system and the inversion algorithm. To this end, the Point Spread Function (PSF) is a powerful and suitable tool for understanding the efficiency of the system because it provides the minimum detail that can be reconstructed. It can be defined as the reconstruction of a point-like scatterer in the spatial domain and the limited resolution can be observed in terms of the PSF of the system.

The PSF behavior analysis is also connected to the Number of Degrees of Freedom (NDF) of the problem, i.e., the number of independent pieces of information that can be reconstructed reliably by an imaging algorithm in the presence of noise on data [5]. The NDF concept has been considered in [6-8] to be used for optical imaging applications and in solving inverse source [9-11] and inverse scattering problems [10,12,13].

The Singular Values Decomposition (SVD) of the relevant operator is the important mathematical tool to provide the NDF. In fact, the NDF is the number of its significant singular values and might be roughly supposed as the number of independent point-like scatterers that can be reconstructed reliably; thus, it can be used to provide the maximum achievable resolution. Based on the SVD properties, the PSF is connected to the number of relevant singular functions of the operator that is the NDF.

One of the most critical criterion for evaluating the efficiency of a radar system is its ability to differentiate between two close objects. The resolution describes this criterion, and in [14] it has been evaluated using a numerical analysis based on the system function. The concept of PSF has been studied in $[15,16]$. For instance, the characterization of the PSF behavior of radially displaced point scatterers for circular synthetic aperture radar has been presented in $[17,18]$.

The PSF concept has been applied in microwave imaging, for instance, for the purposes of comparison of different algorithms $[19,20]$ or near zone observation and half-space geometry [21]; however, either simple Fourier transform arguments within the spectral domain of the far-field data, or numerical results have been employed to perform a resolution analysis.

In [22], we have addressed the PSF analysis of inverse source and scattering problem for strip geometries when the full data are available with the goal of estimating the achievable resolution; furthermore, a valuable approximated PSF, the achievable resolution, and two relevant applications have been introduced. We have considered plane waves impinging on the scattering object from the round angle directions and observation angles along the same round angle. The main conclusion was that the resolution achievable in reconstructing two close point-like scatterers is the same in the whole geometry domain irrespective of the object location for full data. The same analysis for circumference geometries has been performed in [10].

In the present paper, we address the evaluation of the achievable resolution in curve geometries when the number of directions of both the impinging plane waves and the observation angles is limited within an angular domain, giving rise to the so-called aspectlimited problem. It is very common in many practical instances whenever the angular domain around the scattering object is not fully available for sensing purposes as it occurs in many realistic applications such as seismic imaging, Ground-Penetrating Radar (GPR), and subsurface imaging. We adopt the same mathematical tools based on the NDF computation and the PSF evaluation as in the previous papers. In addition, we introduce, and numerically validate an approximated closed-form evaluation of the PSF, so allowing to estimate the resolution in an efficient way. The main result concerns the fact that the resolution changes when the range of plane waves and observation directions vary. For the sake of illustration, the reflection, transmission, and angle modes are introduced to mimic actual sensing configurations. Two numerical examples of the theoretical discussion, concerning two applications both for a Non-Destructive evaluation of a dielectric object of 
known shape and for the localization of point-like scatterers in the presence of uncertainties on the scattering far-field data, are proposed.

This paper is organized as follows. Section 2 introduces the formulation of the problem and the main definitions to be used in the following sections. Section 3 is devoted to the PSF analysis for the circumference geometry. The same analysis is presented in Section 4 for the square geometry. Two numerical applications of the theoretical discussions are provided in Section 5. The discussion and conclusions follow in Section 6.

\section{Problem Statement}

This section aims to provide some mathematical background material to be employed in the following sections. Generally speaking, the relationship between the scattered field and the object parameters is nonlinear. The problem under the Born approximation can be cast as a linear scattering problem.

Figure 1 shows a general view of the inverse scattering problem. An unknown scatterer is located in a domain referred to as the Investigation Domain (ID). We denote the angle defining the direction of propagation of the incident plane wave $E_{i}$ and the observation angle of the scattered far-field $E_{s}$ by $\theta_{i}$ and $\theta_{s}$, respectively. The plane waves domain and observations domain are supposed to be limited so that the range angles of incident plane waves and observations are indicated by $\left[\alpha_{1}, \alpha_{2}\right]$ and $\left[\gamma_{1}, \gamma_{2}\right]$, respectively. It should be noted that the scattering sensing is investigated by changing the incidence angle to improve reconstruction performance and to increase the NDF (multi-view configuration).

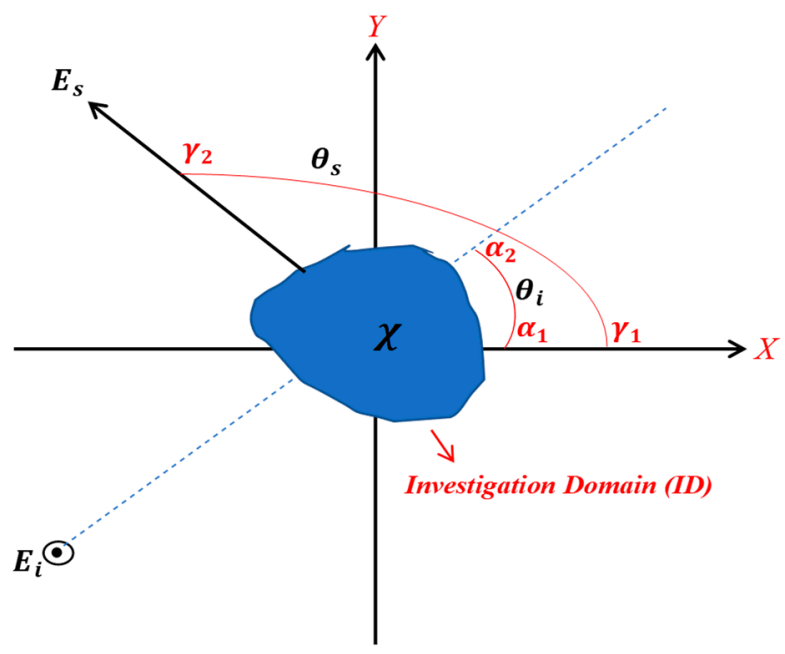

Figure 1. The geometry of the general inverse scattering problem.

The scattered far-field under the Born approximation for the two-dimensional scalar case at a fixed $\omega$ angular frequency is given by

$$
E_{S}\left(\theta_{s}, \theta_{i}\right)=\iint_{I D} \chi(x, y) e^{j \beta\left[x\left(\cos \theta_{s}-\cos \theta_{i}\right)+y\left(\sin \theta_{s}-\sin \theta_{i}\right)\right]} d x d y=\mathcal{T}(\chi(x, y))
$$

apart some inessential factors, where $\chi(x, y)$ and $\mathcal{T}$ are the contrast function and the pertinent linear operator for the multi-view and single frequency scattering configurations of our interest, respectively. An object or scatterer with a relative permittivity of $\epsilon_{\mathcal{S}}(x, y)$, so that $\chi(x, y)=1-\left(\epsilon_{s}(x, y)\right) / \epsilon_{0}$, is located in a homogeneous background, which has a permittivity of $\epsilon_{0}$, where $\epsilon_{0}=8.854 \times 10^{-12} \mathrm{~F} / \mathrm{m}$ is the permittivity of free space. The wavenumber is denoted by $\beta=\omega \sqrt{\left(\epsilon_{0} \mu_{0}\right)}=2 \pi / \lambda$, where, $\mu_{0}=4 \pi \times 10^{-7} \mathrm{H} / \mathrm{m}$ is the magnetic permeability of the free space, and $\lambda$ is the wavelength.

Now, since the $\mathcal{T}$ operator is linear and compact, the SVD can be defined for each scatterer geometry, and the singular system consists of the triple $\left\{v_{n}(x, y), \sigma_{n}, u_{n}(\theta)\right\}$ [23], 
where $u_{n}$ and $v_{n}$ are the singular functions which span the far field data and the object contrast function spaces, respectively, and $\sigma_{n}$ denotes the singular values, arranged under a decreasing order. The adjoint operator of (1) is defined as

$$
\mathcal{T}^{+}\left(E_{S}\right)=\int_{\alpha_{1}}^{\alpha_{2}} \int_{\gamma_{1}}^{\gamma_{2}} E_{\mathcal{S}}\left(\theta_{S}, \theta_{i}\right) e^{-j \beta\left[x^{\prime}\left(\cos \theta_{s}-\cos \theta_{i}\right)+y^{\prime}\left(\sin \theta_{s}-\sin \theta_{i}\right)\right]} d \theta_{\mathcal{S}} d \theta_{i}
$$

Then, the spectral theorem for compact self-adjoint operators are applied to $\mathcal{T}^{+} \mathcal{T}$, it follows that

$$
\mathcal{T}^{+} \mathcal{T}(\chi(x, y))=\iint_{I D} \chi(x, y)\left[\int_{\alpha_{1}}^{\alpha_{2}} \int_{\gamma_{1}}^{\gamma_{2}} e^{j \beta\left[\left(x-x^{\prime}\right)\left(\cos \theta_{s}-\cos \theta_{i}\right)+\left(y-y^{\prime}\right)\left(\sin \theta_{s}-\sin \theta_{i}\right)\right]} d \theta_{s} d \theta_{i}\right] d x d y
$$

whose kernel is

$$
k\left(x-x^{\prime}, y-y^{\prime}\right)=\int_{\gamma_{1}}^{\gamma_{2}} e^{j \beta\left[\left(x-x^{\prime}\right) \cos \theta_{s}+\left(y-y^{\prime}\right) \sin \theta_{s}\right]} d \theta_{s} \cdot \int_{\alpha_{1}}^{\alpha_{2}} e^{-j \beta\left[\left(x-x^{\prime}\right) \cos \theta_{i}+\left(y-y^{\prime}\right) \sin \theta_{i}\right]} d \theta_{i}
$$

Defining

$$
\left\{\begin{aligned}
\widetilde{\rho}=\sqrt{\left(x-x^{\prime}\right)^{2}+\left(y-y^{\prime}\right)^{2}}, \quad \widetilde{\phi} & =\tan ^{-1}\left(\frac{y-y^{\prime}}{x-x^{\prime}}\right) \\
x-x^{\prime}=\widetilde{\rho} \cos \widetilde{\phi}, \quad y-y^{\prime} & =\widetilde{\rho} \sin \widetilde{\phi}
\end{aligned}\right.
$$

then, (4) reads as

$$
k\left(x-x^{\prime}, y-y^{\prime}\right)=\int_{\gamma_{1}}^{\gamma_{2}} e^{j \beta \tilde{\rho} \cos \left(\theta_{s}-\widetilde{\phi}\right)} d \theta_{s} \cdot \int_{\alpha_{1}}^{\alpha_{2}} e^{-j \beta \widetilde{\rho} \cos \left(\theta_{i}-\widetilde{\phi}\right)} d \theta_{i}
$$

The evaluation of (6) is performed as follows. By exploiting the Jacobi-Anger expansion [24], the first factor of (6) is

$$
\int_{\gamma_{1}}^{\gamma_{2}} e^{j \beta \widetilde{\rho} \cos \left(\theta_{s}-\widetilde{\phi}\right)} d \theta_{s}=\sum_{n} j^{n} J_{n}(\beta \widetilde{\rho}) e^{-j n \widetilde{\phi}}\left(\gamma_{2}-\gamma_{1}\right) e^{\frac{1}{2} j n\left(\gamma_{2}+\gamma_{1}\right)} \operatorname{sinc}\left[\frac{n\left(\gamma_{2}-\gamma_{1}\right)}{2}\right]
$$

where $J_{n}(\cdot)$ is the zeroth-order Bessel function of the first kind and $n$-th order and $\operatorname{sinc}(x)=$ $\sin (x) / x$. Then, we compute the second factor of (6) in the same way as

$$
\int_{\alpha_{1}}^{\alpha_{2}} e^{-j \beta \tilde{\rho} \cos \left(\theta_{i}-\widetilde{\phi}\right)} d \theta_{i}=\sum_{m}(-j)^{m} J_{m}(\beta \widetilde{\rho}) e^{j m \widetilde{\phi}}\left(\alpha_{2}-\alpha_{1}\right) e^{\frac{-1}{2} j m\left(\alpha_{2}+\alpha_{1}\right)} \operatorname{sinc}\left[\frac{m\left(\alpha_{2}-\alpha_{1}\right)}{2}\right]
$$

Finally, substituting (7) and (8) into (6), it results

$$
\begin{gathered}
k\left(x-x^{\prime}, y-y^{\prime}\right)=\sum_{\mathrm{n}} j^{n} J_{n}(\beta \widetilde{\rho}) e^{-j n \widetilde{\phi}}\left(\gamma_{2}-\gamma_{1}\right) e^{\frac{1}{2} j n\left(\gamma_{2}+\gamma_{1}\right)} \operatorname{sinc}\left[\frac{n\left(\gamma_{2}-\gamma_{1}\right)}{2}\right] . \\
\sum_{m}(-j)^{m} J_{m}(\beta \widetilde{\rho}) e^{j m \widetilde{\phi}}\left(\alpha_{2}-\alpha_{1}\right) e^{\frac{-1}{2} j m\left(\alpha_{2}+\alpha_{1}\right)} \operatorname{sinc}\left[\frac{m\left(\alpha_{2}-\alpha_{1}\right)}{2}\right]
\end{gathered}
$$

The PSF is now considered to evaluate the performance of the reconstruction algorithm. The PSF analysis is performed in the inverse scattering problem to understand how the scatterer geometry and the aspect-limited available data may affect the resolution. The PSF represents the reconstruction of a point-like scatterer. The aim is to obtain an analytical estimation for achievable resolution and to connect it to the geometrical parameters. To this end, we start to consider the PSF for a general 2D scatterer and then apply it to the other scatterer geometries.

Two main questions arise when the limited views and observations are considered. The first is to find the NDF and how it can be changed for aspect-limited data. The second is to estimate the achievable resolution and to show how it can be varied when the position of the point scatterer changes.

As far as the first point is concerned, as it is not easy to perform an analytical evaluation, the numerical evaluation of the SVD of the pertinent operator provides the NDF.

On the other hand, the PSF is observed at $(x, y)$ when the point-like scatterer is located at $\left(x_{0}, y_{0}\right)$. From the mathematical point of view, the PSF in the scatterer domain is defined 
as the impulsive response of the system provided by the cascade of the $\mathcal{T}^{-1}$, i.e., the regularized inverse operator of $\mathcal{T}$, and the direct operator, as

$$
\operatorname{PSF}\left(x, x_{0}, y, y_{0}\right)=\left(\mathcal{T}^{-1} \mathcal{T}\right) \delta\left(x-x_{0}\right) \delta\left(y-y_{0}\right)
$$

where $\delta$ is the Dirac delta-function.

Thus, since the minimum-norm solution of the inverse scattering problem is the projection of the actual contrast function onto the singular function $v_{n}$ having non-zero singular values, the PSF for the inverse scattering problem is given by the completeness relation truncated to the singular functions having non-zero singular values; therefore the PSF is dependent on the number of retained singular values, which, in turn, is connected to the noise level. Then, if a truncated SVD (TSVD) [23] inversion scheme is adopted to implement the regularized inversion step, thus Equation (10) can be written explicitly as

$$
\operatorname{PSF}\left(x, x_{0}, y_{1} y_{0}\right)=\sum_{n=1}^{N} v_{n}(x, y) v_{n}^{*}\left(x_{0}, y_{0}\right)
$$

where $*$ means conjunction operation and $N$ is a truncation index, which can be estimated as the knee of the singular values curve. Therefore, the $N$ value can be selected equal to the NDF.

Equation (11) requires the knowledge of the singular functions, which can be computed in closed form only for limited instances of scatterer geometries. On the other hand, if the adjoint operator may approximate the inverse operator in Equation (11), it can be considered to build a good approximation of the PSF [10,22]. This occurs when the singular values of the scattering operator exhibit a nearly constant behavior before the knee of their curve. Consequently, by replacing the adjoint operator instead of the inversion operator in (10), we define the approximated $\widetilde{P S F}$ by

$$
\widetilde{\operatorname{PSF}}\left(x, x_{0}, y, y_{0}\right)=\left(\mathcal{T}^{+} \mathcal{T}\right) \delta\left(x-x_{0}, y-y_{0}\right)
$$

Accordingly, Equation (9) provides the analytical evaluation of (12) for the 2D geometry under consideration.

\section{Circumference Geometry}

In this section, we address a dielectric circumference, as shown in Figure 2. Its radius is indicated by $\rho$. The reflection, transmission, and angle modes are introduced to provide the effect of each mode on NDF and the achievable resolution. Furthermore, the approximated $\widetilde{P S F}$ is derived from the above general case to compare with the actual PSF.

The scattered far-field of the considered geometry is given by

$$
E_{s c}\left(\theta_{S}, \theta_{i}\right)=\int_{I D} \chi_{\mathcal{c}}(\phi) e^{j \beta \rho\left[\cos \phi\left(\cos \theta_{s}-\cos \theta_{i}\right)+\sin \phi\left(\sin \theta_{s}-\sin \theta_{i}\right)\right]} \rho d \phi=\mathcal{T}_{c}\left(\chi_{\mathcal{c}}(\phi)\right)
$$

By replacing $\widetilde{\rho}_{c}$ and $\widetilde{\phi_{c}}$

$$
\left\{\begin{array}{c}
\widetilde{\rho}_{c}=2 \rho \sin \left(\frac{\phi-\phi^{\prime}}{2}\right) \\
\tan \widetilde{\phi}_{c}=-\tan \left(\frac{\phi+\phi^{\prime}}{2}\right)
\end{array}\right.
$$

instead of $\widetilde{\rho}$ and $\widetilde{\phi}$ in (6), Equation (9) provides the analytical evaluation of the kernel of $\mathcal{T}_{c}^{+} \mathcal{T}_{c}$ that is the analytical approximated $\widetilde{P S F}$. 


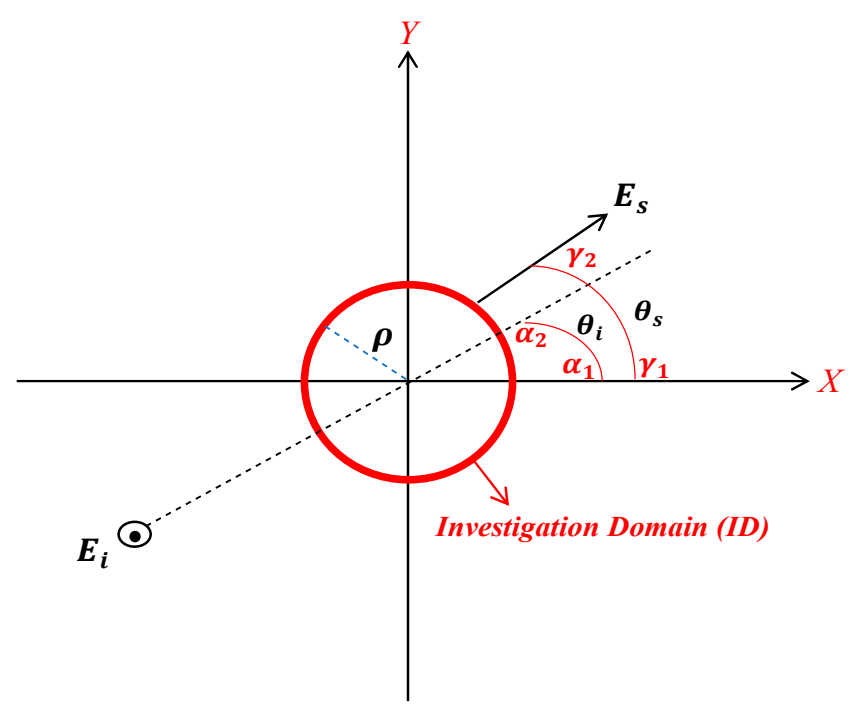

Figure 2. The geometry of the circumference ID.

Hereafter we consider three possibilities for the combination of impinging angles of the plane waves (transmitter) and observation directions (receiver) for the aspect limited data case. If the transmitters and the receivers are co-located, we call it the reflection mode. For instance, the back-scattered fields are only available when the transmitters and receivers are on the same side as it occurs in a GPR application. Conversely, the transmission mode is when the receivers are separate and located at the opposite side of the transmitters. For instance, the forward scattered fields are only available if the transmitters and receivers are on different sides, as boreholes in seismic prospection. Finally, the angle mode is defined when the transmitters and the receivers are located on adjacent sides. It may occur in Through-the-Wall Imaging applications when transmitters and receivers are separated and located close to two contiguous walls of a room.

Some numerical simulations are presented to confirm the analytical discussions and to compare the achievable resolution in the reflection mode with the transmission mode. Throughout the paper, to this end, we employ a sufficiently fine discretization of the relevant integral Equation (1) to compute the SVD of the resulting matrix equation under the MATLAB environment. Let consider the geometry as shown in Figure 2. We consider that the radius of the circumference is $\rho=3 \lambda$. The angular range of the impinging plane waves and observation directions is $\pi / 2$ wide so that $\alpha_{1}$ and $\alpha_{2}$ are equal to $-\pi / 4$ and $\pi / 4$, respectively. Furthermore, the angular range of the observation directions is $\pi / 2$ wide that $\gamma_{1}=3 \pi / 4$ and $\gamma_{2}=5 \pi / 4$ when the sensing system is in the reflection mode, $\gamma_{1}=-\pi / 4$ and $\gamma_{2}=\pi / 4$ in the transmission mode, and $\gamma_{1}=\pi / 4, \gamma_{2}=3 \pi / 4$ in the angle mode, respectively.

We point out that the NDF of the circumference geometry has been computed analytically for the general full-view case in [10], resulting in 75, much larger than the corresponding value of NDF of the present aspect limited case, which is computed numerically and is around 30 for all three modes. A comparison between the behavior of the singular values of three modes is shown in Figure 3.

Generally, the width of the main lobe of the PSF is connected to the resolution in inverse scattering problems, that is, the capability of the inversion scheme to distinguish reliably two close scatterer points. While from $[10,22]$ a PSF invariant with the point-like scatterer position is predicted analytically, in the present aspect limited case, this is not the case as (9) does not depend only on the angular difference $\phi-\phi_{0}$. This means that, in principle, all scatterer points cannot be imaged in the same way, independent of their positions. 
Generally speaking, since the modulus of the $\widetilde{P S F}$ f for both the transmission and the reflection modes are equal by (9), we expect that this result holds true also for the actual ones.

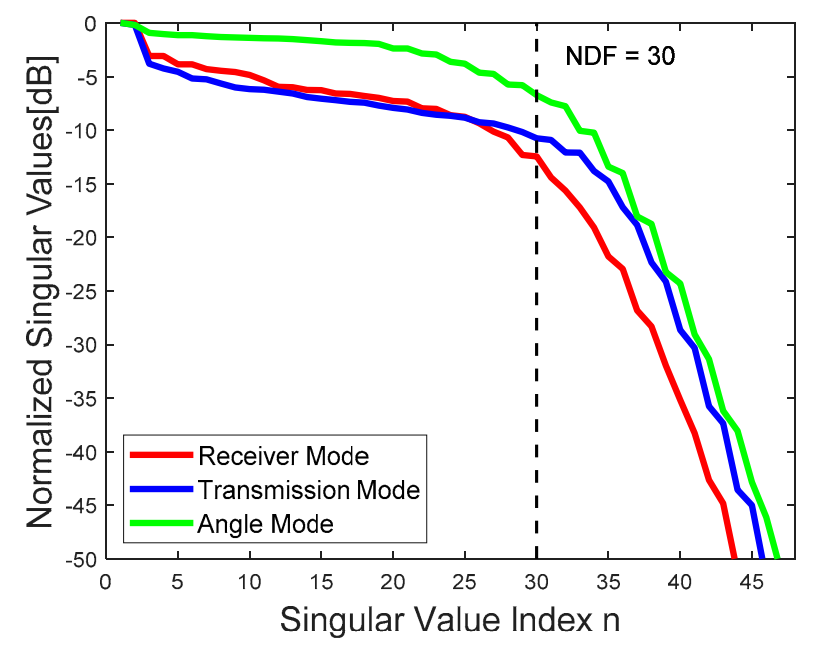

Figure 3. Comparison of the behavior of the normalized singular values of the relevant operators for reflection, transmission, and angle modes.

A comparison of the actual PSFs of the three modes is provided in Figures 4 and 5. The considered point positions are $\phi_{0}=0, \pi / 6, \pi / 4$ (Figure 4 ) and $\phi_{0}=\pi / 2,5 \pi / 6, \pi$ (Figure 5) respectively, and the abscissa axis of the figures is provided in terms of the arc length over the circumference to point out the resolution in terms of the distance variable by means of the Full-Width Half Maximum (FWHM) criterion.

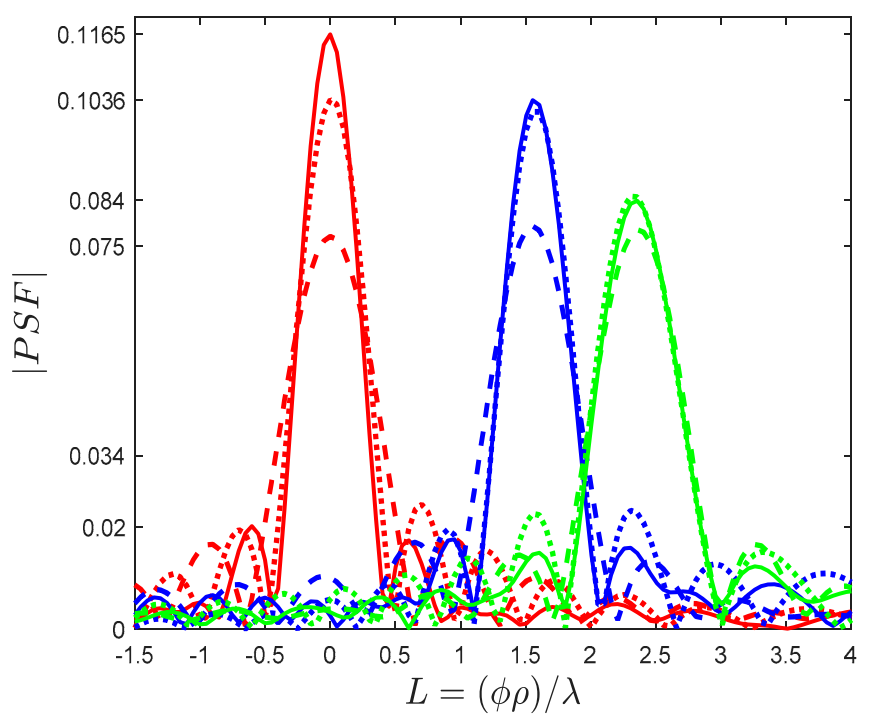

Figure 4. Comparison of the actual PSFs of three sensing modes for $\phi_{0}=0$ (red lines), $\phi_{0}=\pi / 6$ (blue lines), and $\phi_{0}=\pi / 4$ (green lines). The solid, dotted, and dashed lines indicate the reflection, transmission, and angle modes.

In the reflection and transmission modes, if the location of the scatterer point changes, the width of the main lobe changes as well, i.e., it is spatially variant, and consequently, the resolution is not constant. However, it can be observed that for the considered geometry, it is approximately the same, at about $0.5 \lambda$, with slight differences at about $0.05 \lambda$; when the scatterer point is located at the edge or outside the angular range of the plane wave and observation domains, the width of the main lobe of $\phi_{0}=\pi / 2$ is significantly larger than 
the others. Interestingly, the PSF result is approximately the same for each point at ID in the angle mode at about $0.65 \lambda$, which means the resolution does not change appreciably and it is approximately space invariant; in addition, it is always slightly larger than the one for both transmission and reflection modes.

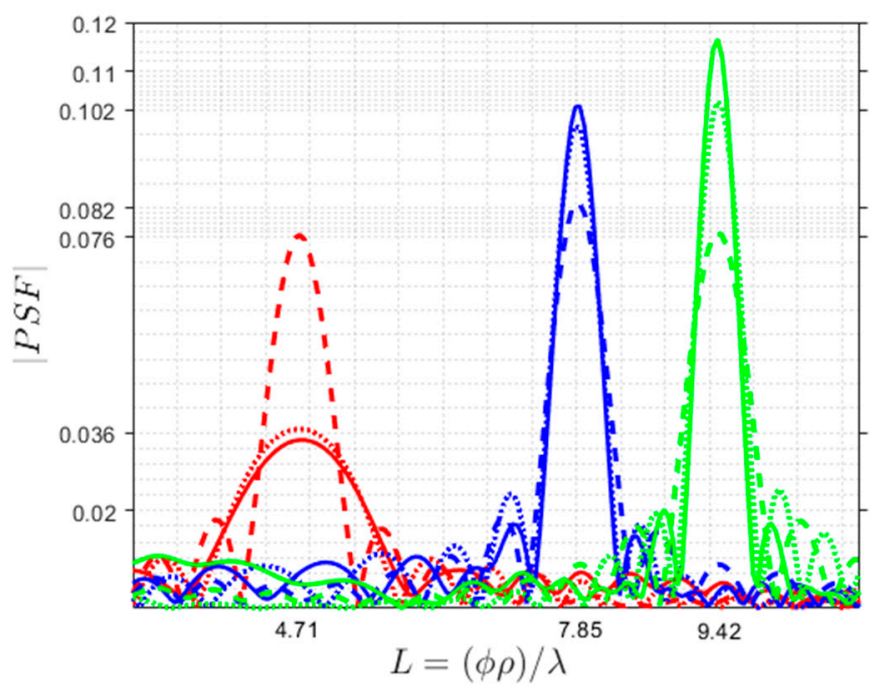

Figure 5. Comparison of the actual PSFs of three sensing modes for $\phi_{0}=\pi / 2$ (red lines), $\phi_{0}=5 \pi / 6$ (blue lines), $\phi_{0}=\pi$ (green lines). The solid, dotted, and dashed lines indicate the reflection, transmission, and angle modes.

In order to confirm the performance of the approximated $\widetilde{P S F}$, a comparison between it and the actual PSF for three sensing modes is provided, as shown in Figure 6. Since we are only interested in the main lobe of the $\widetilde{P S F}$ we show only it. The amplitude of both PSFs normalized to 1 . The numerical example verifies that the approximation is acceptable, at least in the main lobe. As can be observed, the approximation does not overlap exactly with the actual in the $\phi_{0}=\pi / 2$ in the reflection and transmission modes because the point is located outside of the observation ranges. Its performance can improve when the scatterer point is located inside the ranges. Consequently, the plane wave directions and observation directions are important for reconstructing the point-like scatterer. Therefore, we can conclude that the resolution depends on the position of the scatterer point and on the adopted sensing mode.
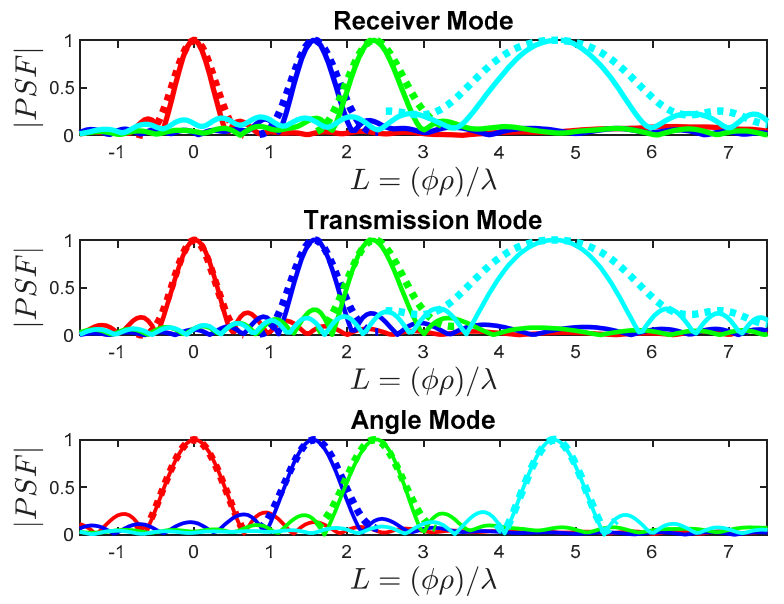

Figure 6. Comparison between the actual PSF (solid lines) and the approximation $\widetilde{P S F}$ (dotted lines) for three modes for $\phi_{0}=0$ (red lines), $\phi_{0}=\pi / 6$ (blue lines), $\phi_{0}=\pi / 4$ (green lines) and $\phi_{0}=\pi / 2$ (cyan lines). 


\section{Square Geometry}

This section deals with the same analysis as in Section 3 to address a square consisting of four connected strips, as shown in Figure 7.

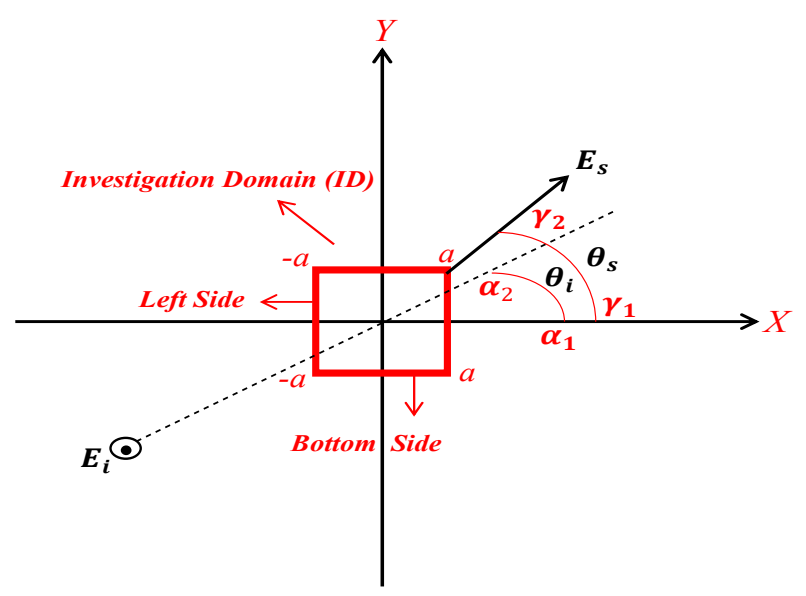

Figure 7. The geometry of the square ID.

The scattered far-field is provided by (1) when $(x, y) \in I D$ and the integral is now one-dimensional, whereas the corresponding adjoint operator is provided by (2) with $\left(x^{\prime}, y^{\prime}\right) \in I D$. The resulting approximate PSF for the horizontal sides require to compute

$$
\begin{aligned}
& \widetilde{P S F}_{h}\left(x-x^{\prime}\right)=\int_{\gamma_{1}}^{\gamma_{2}} e^{j \beta\left[\left(x-x^{\prime}\right) \cos \theta_{s}\right]} d \theta_{s} \cdot \int_{\alpha_{1}}^{\alpha_{2}} e^{-j \beta\left[\left(x-x^{\prime}\right) \cos \theta_{i}\right]} d \theta_{i}= \\
& \sum_{n} j^{n} J_{n}\left(\beta\left(x-x^{\prime}\right)\right)\left(\gamma_{2}-\gamma_{1}\right) e^{\frac{1}{2} j n\left(\gamma_{2}+\gamma_{1}\right)} \operatorname{sinc}\left[\frac{n\left(\gamma_{2}-\gamma_{1}\right)}{2}\right] . \\
& \sum_{m}(-j)^{m} J_{m}\left(\beta\left(x-x^{\prime}\right)\right)\left(\alpha_{2}-\alpha_{1}\right) e^{\frac{-1}{2} j m\left(\alpha_{2}+\alpha_{1}\right)} \operatorname{sinc}\left[\frac{m\left(\alpha_{2}-\alpha_{1}\right)}{2}\right]
\end{aligned}
$$

For the vertical strips, we require

$$
\begin{aligned}
& \widetilde{P S F}_{v}\left(y-y^{\prime}\right)=\int_{\gamma_{1}}^{\gamma_{2}} e^{j \beta\left[\left(y-y^{\prime}\right) \sin \theta_{s}\right]} d \theta_{s} \cdot \int_{\alpha_{1}}^{\alpha_{2}} e^{-j \beta\left[\left(y-y^{\prime}\right) \sin \theta_{i}\right]} d \theta_{i}= \\
& \sum_{n} J_{n}\left(\beta\left(y-y^{\prime}\right)\right)\left(\gamma_{2}-\gamma_{1}\right) e^{\frac{1}{2} j n\left(\gamma_{2}+\gamma_{1}\right)} \operatorname{sinc}\left[\frac{n\left(\gamma_{2}-\gamma_{1}\right)}{2}\right] . \\
& \sum_{m} J_{m}\left(\beta\left(y-y^{\prime}\right)\right)\left(\alpha_{2}-\alpha_{1}\right) e^{\frac{-1}{2} j m\left(\alpha_{2}+\alpha_{1}\right)} \operatorname{sinc}\left[\frac{m\left(\alpha_{2}-\alpha_{1}\right)}{2}\right]
\end{aligned}
$$

Equations (15) and (16) give the analytical of the approximated $\widetilde{P S F}$ for the horizontal and vertical strips, respectively. They show that the $\widetilde{P S F S}$ depend only on the distance between the source and the current points along each side, thus implying a constant resolution and spatial invariant behavior. However, the resolution may differ on different sides of the present ID.

In the following numerical simulations, $a=3 \lambda$ is considered for the reflection, transmission, and angle modes. The ranges $\left[\alpha_{1}, \alpha_{2}\right]$ and $\left[\gamma_{1}, \gamma_{2}\right]$ are the same as in the previous section. Figure 8 presents the behavior of singular values of the relevant operators for three modes to compute the NDF numerically. The geometry of the problem is depicted in Figure 7 . By following the strategy of $[9,13]$, the whole NDF of the square can be provided approximately by summing the NDF of each strip, and the NDF of full view is equal to 96 . The numerical results for the aspect limited case show that the NDF of the three modes is approximately equal to 38 , and the only difference between them concerns the behavior of the singular values.

A comparison between the actual PSF of the bottom side of the three modes for three points located at $x_{0}=-1.5 \lambda, 0 \lambda, 2 \lambda$ are plotted in Figure 9. We observe that the PSF results of the reflection and transmission modes are the same, as already observed in the previous section. Moreover, if the location of the scatterer point changes, the width of 
the main lobe does not change; however, the amplitude of the main lobe varies, which means the resolution remains constant. Nevertheless, the PSF results in the angle mode are different for considered points compared to other modes.

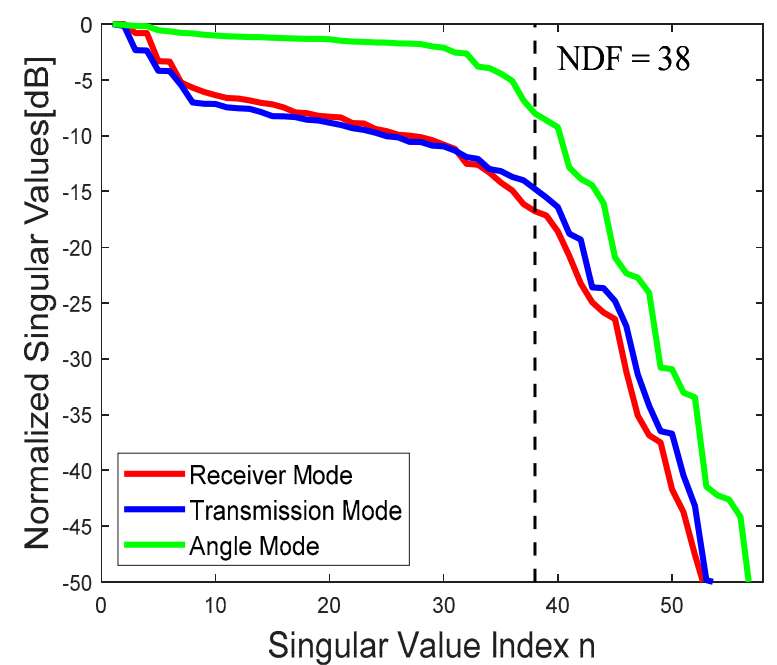

Figure 8. Comparison of the behavior of the normalized singular values of the reflection mode (red line), transmission mode (blue line), and angle mode (green line).

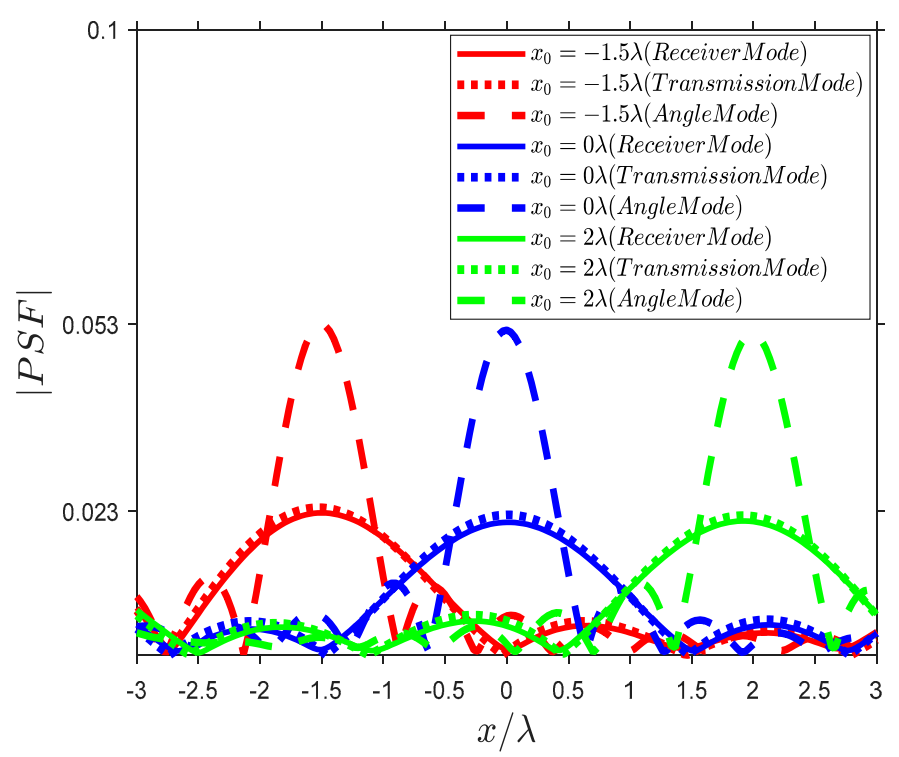

Figure 9. Comparison between the actual PSFs of the bottom side for three different points in the three modes.

It is noteworthy that if the scatterer point locates outside of the range of the plane wave, the width of the main lobe is broad, its amplitude is small, and it will be difficult to reconstruct it accurately. This feature is significant, and it can be used in applications, for instance, when a specific part of the scatterer needs to be reconstructed. Consequently, in order to reconstruct the required side of the scatterer satisfactorily, the position of transmitters and receivers should be selected adequately.

The actual PSFs of the left side for three points $y_{0}=-1.5 \lambda, 0 \lambda, 2 \lambda$ in the three modes are addressed to present the difference between them, as shown in Figure 10. It is observed that when the position of the point scatterer changes, the resolution will be constant; however, the amplitude of the main lobe varies slightly. There is no remarkable difference between the PSFs of the reflection and transmission modes, although, in the angle mode, the width of the main lobe is broader. 


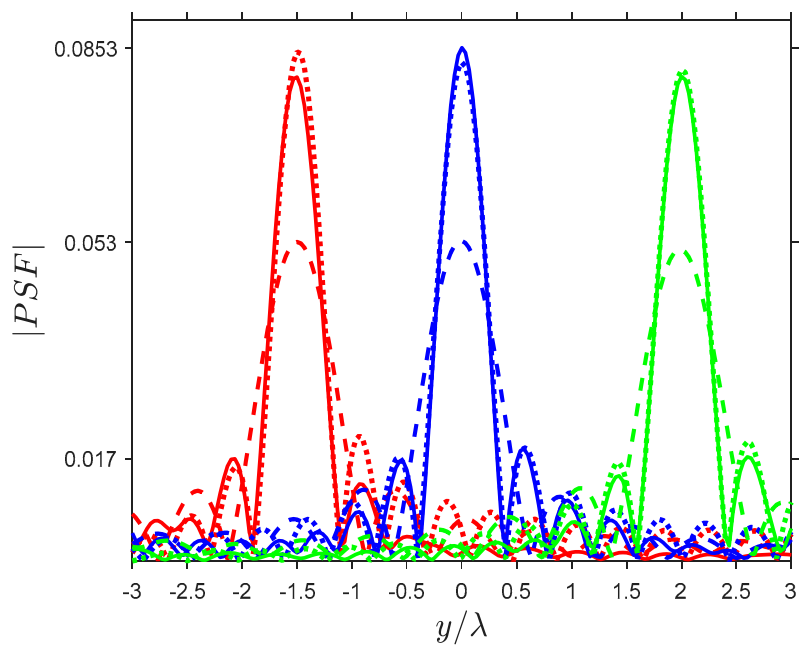

Figure 10. Comparison between the actual PSFs of the left side for $x_{0}=-1.5 \lambda$ (red lines), $x_{0}=0 \lambda$ (blue lines), and $x_{0}=2 \lambda$ (green lines) in reflection mode (solid lines), transmission mode (dotted lines), and angle mode (dashed lines).

By comparing the results of both sides, the amplitude of the PSF of the left side is larger than the bottom side in the reflection and transmission modes; however, the width of the left side is narrower than the bottom side. It can be concluded that the amplitude of the PSF and resolution are different for each side of the square; moreover, they depend on the range of transmitter and receiver directions. The space-variant behavior of the PSF only occurs when the scatterer point moves from the bottom side to the left side of the square in the reflection and transmission modes. We again observe that the PSF result is the same for each side in the angle mode, which means the resolution is unchanged on each side, as it can be forecast by the analytical behavior of (15) and (16).

Three comparisons between the actual PSF and approximated $\widetilde{P S F}$ for the bottom and left sides in the three modes are shown in Figures 11-13. To this end, we consider only the main lobe of the $\widetilde{P S F}$ to outline the focusing properties. As shown in Figures 11 and 12, the main lobe of the $\widetilde{P S F}$ of the bottom side is not overlapped when the scatterer point is not located within the plane waves and observations angular ranges, and the approximation is acceptable therein. Nonetheless, the results of Figure 13 confirm that the approximation works well in the main lobe for both sides to predict the achievable resolution for every scatterer point.
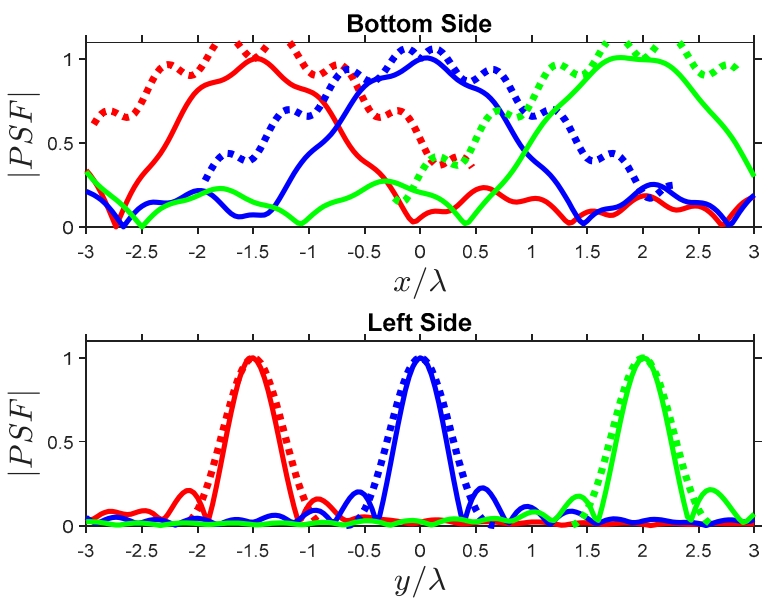

Figure 11. Comparison of the actual PSF (dotted lines) and approximated $\widetilde{P S F}$ (solid lines) for the bottom and left sides in the reflection mode for $x_{0}=-1.5 \lambda$ (red lines), $x_{0}=0 \lambda$ (blue lines), and $x_{0}=2 \lambda$ (green lines). 

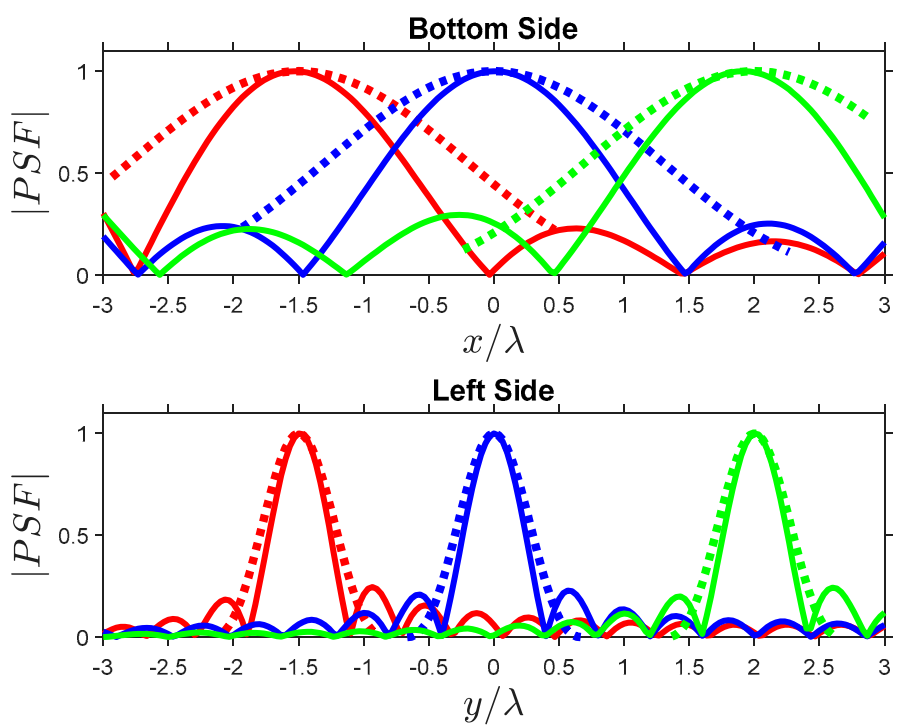

Figure 12. Comparison of the actual PSF (dotted lines) and approximated $\widetilde{P S F}$ (solid lines) for the bottom and left sides in the transmission mode for $x_{0}=-1.5 \lambda$ (red lines), $x_{0}=0 \lambda$ (blue lines), and $x_{0}=2 \lambda$ (green lines).
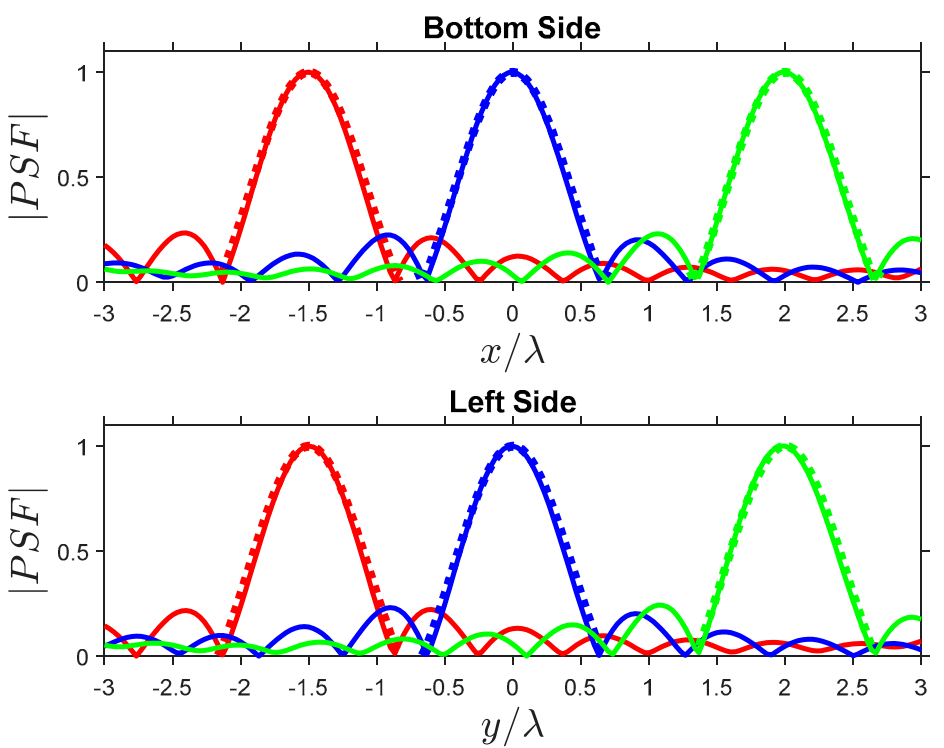

Figure 13. Comparison of the actual PSF (dotted lines) and approximated $\widetilde{P S F}$ (solid lines) for the bottom and left sides in the angle mode for $x_{0}=-1.5 \lambda$ (red lines), $x_{0}=0 \lambda$ (blue lines), and $x_{0}=2 \lambda$ (green lines).

\section{Numerical Applications}

This section is devoted to introducing two relevant applications of the above theoretical discussions and, furthermore, to providing the effects of the incidence and observation ranges on the reconstruction. They can be used for recovering unknown object information from the knowledge of aspect limited far-field data.

Resolution limits in reconstructions are commonly introduced by means of the Rayleigh criterion as the minimum detectable separation between two close scatterer points. It can be appreciated by referring to the degree of overlap of the main lobes of the corresponding PSFs pattern. Two points are generally resolved if the first maximum of one PSF falls into the first minimum of the other PSF, but there is no standard definition, especially when the resolution is not constant. In [22], we applied this criterion for strip geometries when the full data is available. 
The first application concerns the reconstruction of cracks within a dielectric square located in free space with $a=3 \lambda, \lambda / 10$ large, as illustrated in Figure 14. The background is the free space, and the contrast between the scatterer and the background is $\chi=1$. The scattered field data are assumed to be collected in the far zone at a distance with a radius larger than $36 \lambda$.

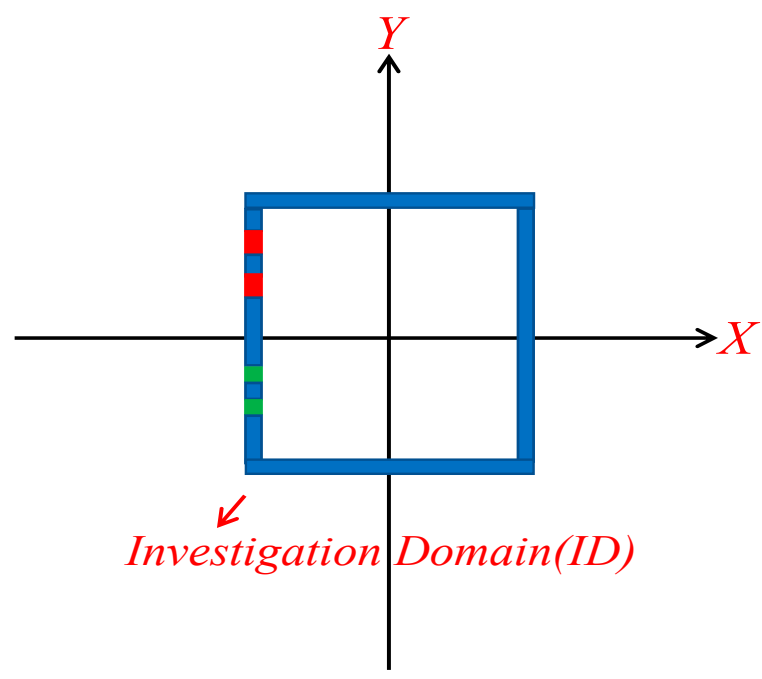

Figure 14. The geometry of the dielectric object (blue) with cracks (red and green).

In this section, we consider the reflection mode for reconstructions. By using the results in Section 4, the achievable resolution of the left side is expected to be equal to $0.4 \lambda$ at the chosen position; thus, the size of the cracks and the distance between two cracks is selected with reference to the resolution. The reconstruction is only considered for the left side because the resolution of the bottom side is very low. Therefore, we suppose that two cracks (free space condition, $\chi=0$ ) lay on the left side (denoted by the red lines), being $0.4 \lambda$ both the length of each crack and the distance between them; furthermore, two more cracks (green lines) also exist on the same side, being $0.3 \lambda$ both the size of each crack and the distance between them.

Firstly, the scattered far-field data are only observed over a limited range of angles as the same in the previous sections. Then, an additive white Gaussian noise is added to the simulated scattered field, with a noise level such that the Signal-to-Noise Ratio (SNR) is $10 \mathrm{~dB}$. Finally, the Truncated SVD algorithm is adopted to reconstruct the contrast function, and the threshold value is fixed in terms of NDF to mitigate the effect of noise. The computed NDF $=38$ are provided in Section 4 .

The reconstructed results of the left side of the square are shown in Figure 15. It can be distinguished that two cracks are present along the left side when their size and the distance between them are equal to the resolution, and they can also be resolved well in the presence of noise on data. On the contrary, it is impossible to discriminate between two cracks when their sizes and distance are smaller than the resolution. Hence, this testifies that a preliminary resolution analysis is important for the reliable reconstruction of defects in dielectric objects in linear inverse scattering problems.

The second example concerns the reconstruction of two close point-like scatterers, mimicking a localization problem (f.i. two bodies for a surveillance radar application). Since the PSF is a space-variant function in the reflection mode, which means the reconstruction of a scatterer point depends on its location, leading to different resolution limits, it is necessary to examine the case where the scattering points are nonuniformly located along with ID. 


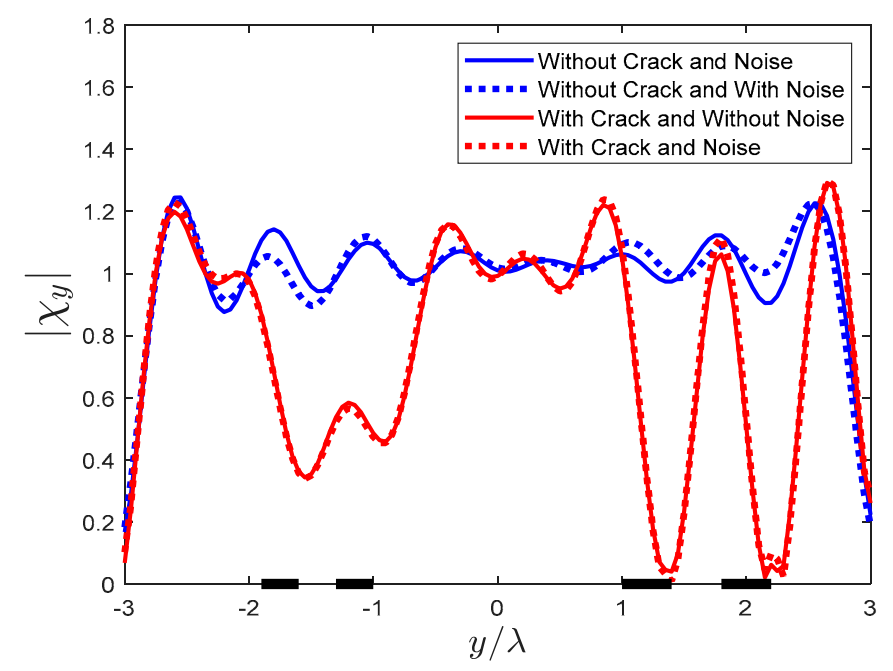

Figure 15. The reconstruction of the contrast function on the left side of the square in the presence of noise. The bold black lines indicate the position of the cracks.

Figure 16 shows the result for point-like objects located on a circumference with $\rho=3 \lambda$. The scattered field data are assumed to be collected in the far zone at a distance with a radius larger than $18 \lambda$. The results of Section 3 are here used as this reconstruction comes directly from the summation of the PSFs centered at the corresponding points; accordingly, in Figure 16, we compare the summations of the exact PSFs with the ones of the $\widetilde{P S F}$, in order to point out the usefulness of the analysis of Section 3 about the availability of closed-form PSFs. The amplitude of both PSFs normalized to 1 . We consider a couple of points at $\phi_{0}=0, \pi / 12, \phi_{0}=\pi / 3,5 \pi / 12$ and is $\phi_{0}=2 \pi / 3,3 \pi / 4$ so that the distance between them is always $L=0.78 \lambda$, corresponding to the resolution distance according to the Rayleigh criterion for the first couple of points.

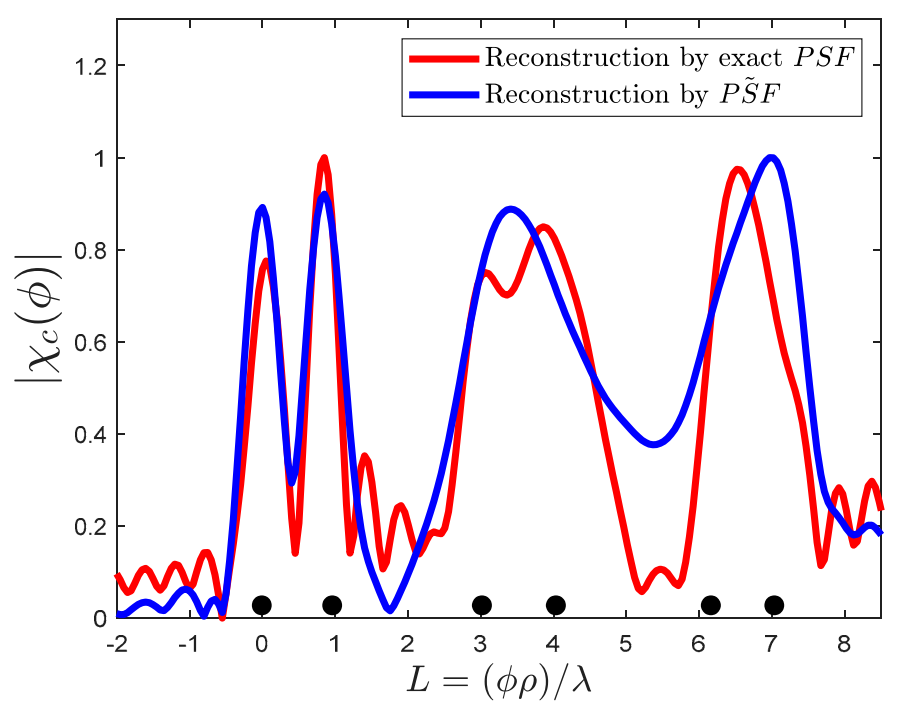

Figure 16. The reconstructions of some point-like scatterer by both actual and approximated PSFs. The black dots indicate the position of the scatterer points.

As it is observed in Figure 16, it is possible to distinguish between the two scattering points only for the first couple, which is spaced the correct resolution distance. Conversely, the result looks like the reconstruction of a single scattering point for the other couples of points, whose resolution distance is larger. Consequently, the scattering system under aspect limited excitations and observations fails to differentiate between two close points. 
Therefore, like the results of Section 3, the effect of the incidence and observation ranges on the resolution of two close point-like scattering objects can be predicted by comparing the reconstructions of [10] when the full data is available and a satisfactory reconstruction can be obtained even when the incidence and observation ranges do not span the round angle. However, we can only obtain a lower accurate reconstruction and, moreover, it is impossible to reconstruct point-like objects located on the whole ID with the same accuracy.

\section{Discussion and Conclusions}

The resolution analysis in linear inverse scattering problems allows to appreciate the performances of imaging algorithms as far as the capability of localizing point-like objects is concerned. In this paper, we keep on this goal by investigating the role of the NDF and the PSF as derived by SVD of the relevant scattering operator. We consider simple investigation geometries (like a circumference and a square) in order to keep at a minimum the computational burden and to be able to achieve close form results whenever possible. In particular, the novelty of the paper consists in the examination of the case of aspect-limited data, namely when the plane waves impinging from a limited angular range and the observation domain scans a limited domain in the far zone region.

While the PSF can be obtained by the numerical solution of the relevant linear inverse problem, an approximate analytical evaluation is introduced, and its accuracy is assessed for each geometry by numerical simulations. A very good agreement is found in the main lobe region of both PSFs, which is far enough to predict the actual resolution of a sensing configuration. The only exception occurs for the case of point-like scatterers located outside the plane wave and observation angle ranges.

The main practical result of the theoretical analysis concerns the fact that the resolution changes as the position of the scatterer point varies when the incidence and observation angular domains are limited. Three different sensing modalities, called the reflection, transmission, and angle modes, are introduced to show their difference in the NDF and resolution. For the considered numerical examples, the results show that the NDF is approximately the same for all modes; on the contrary, the resolution is not the same over the investigation domain.

Finally, the resolution analysis is applied to two numerical examples concerning the detection of cracks within dielectric for Non-Destructive Testing and the localization of point-like scatterers (mimicking two bodies) in the presence of uncertainties on the scattering far-field data for a surveillance radar imaging system.

The paper results confirm the possibility of forecasting the resolution in the reconstructions by the availability of closed-form analytical PSFs; this may be very helpful in comparing different sensing modalities in the common practical conditions of aspect limited data. The whole analysis is currently under extension to the full $2 \mathrm{D}$ and $3 \mathrm{D}$ geometries.

Author Contributions: Conceptualization, G.L. and R.P.; methodology, G.L. and R.P.; software, E.A.S.; validation, E.A.S. and G.L.; formal analysis, E.A.S. and G.L.; investigation, E.A.S. and G.L.; resources, E.A.S.; data curation, E.A.S.; writing-original draft preparation, E.A.S.; writing-review and editing, G.L.; visualization, G.L.; supervision, R.P.; project administration, G.L.; funding acquisition, R.P. All authors have read and agreed to the published version of the manuscript.

Funding: This research received no external funding.

Conflicts of Interest: The authors declare no conflict of interest.

\section{References}

1. Ahn, C.Y.; Jeon, K.; Ma, Y.-K.; Park, W.-K. A study on the topological derivative-based imaging of thin electromagnetic inhomogeneities in limited-aperture problems. Inverse Probl. 2014, 30, 105004. [CrossRef]

2. Bao, G.; Liu, J. Numerical Solution of Inverse Scattering Problems with Multi-experimental Limited Aperture Data. SIAM J. Sci. Comput. 2003, 25, 1102-1117. [CrossRef] 
3. Mager, R.D.; Bleistein, N. An Approach to the Limited Aperture Problem of Physical Optics Farfield Inverse Scattering; Tech. Rep.; University of Denver: Denver, CO, USA, 1976.

4. Zinn, A. On an optimisation method for the full- and the limited-aperture problem in inverse acoustic scattering for a sound-soft obstacle. Inverse Probl. 1989, 5, 239-253. [CrossRef]

5. Piestun, R.; Miller, D.A.B. Electromagnetic degrees of freedom of an optical system. J. Opt. Soc. Am. A 2000, 17, 892-902. [CrossRef] [PubMed]

6. Miller, D.A.B. Communicating with waves between volumes: Evaluating orthogonal spatial channels and limits on coupling strengths. Appl. Opt. 2000, 39, 1681-1699. [CrossRef] [PubMed]

7. Di Francia, G.T. Degrees of Freedom of an Image. J. Opt. Soc. Am. 1969, 59, 799-803. [CrossRef] [PubMed]

8. Bendinelli, M.; Consortini, A.; Ronchi, L.; Frieden, B.R. Degrees of freedom, and eigenfunctions, for the noisy image. J. Opt. Soc. Am. 1974, 64, 1498-1502. [CrossRef]

9. Sekehravani, E.A.; Leone, G.; Pierri, R. NDF of the far zone field radiated by square sources. In Proceedings of the 2021 XXXIVth General Assembly and Scientific Symposium of the International Union of Radio Science, URSI GASS, Rome, Italy, 28 August-4 September 2021; pp. 1-4.

10. Sekehravani, E.A.; Leone, G.; Pierri, R. NDF and PSF analysis in inverse source and scattering problems for circumference geometries. Electronics 2021, 10, 2157. [CrossRef]

11. Solimene, R.; Pierri, R. Number of degrees of freedom of the radiated field over multiple bounded domains. Opt. Lett. 2007, 32, 3113-3115. [CrossRef] [PubMed]

12. Bertero, M.; Viano, G.; Pasqualetti, F.; Ronchi, L.; Di Francia, G.T. The Inverse Scattering Problem in the Born Approximation and the Number of Degrees of Freedom. Opt. Acta: Int. J. Opt. 1980, 27, 1011-1024. [CrossRef]

13. Akbari Sekehravani, E.; Leone, G.; Pierri, R. NDF of scattered fields for strip geometries. Electronics 2021, 10, 202. [CrossRef]

14. Brancaccio, A.; Leone, G.; Pierri, R. Information content of Born scattered fields: Results in the circular cylindrical case. J. Opt. Soc. Am. A 1998, 15, 1909-1917. [CrossRef]

15. Solimene, R.; Maisto, M.A.; Pierri, R. Inverse scattering in the presence of a reflecting plane for the strip case. J. Opt. 2015, 18, 025603. [CrossRef]

16. Rihaczek, A. Radar resolution of ideal point scatterers. IEEE Trans. Aerosp. Electron. Syst. 1996, 32, 842-845. [CrossRef]

17. Solimene, R.; Leone, G.; Pierri, R. Resolution in two-dimensional tomographic reconstructions in the Fresnel zone from Born scattered fields. J. Opt. A Pure Appl. Opt. 2004, 6, 529-536. [CrossRef]

18. Maisto, M.A.; Solimene, R.; Pierri, R. Depth resolution in strip current reconstructions in near non-reactive zone. J. Opt. Soc. Am. A 2019, 36, 975-982. [CrossRef] [PubMed]

19. Catapano, I.; Soldovieri, F.; Alli, G.; Mollo, G.; Forte, L.A. On the Reconstruction Capabilities of Beamforming and a Microwave Tomographic Approach. IEEE Geosci. Remote. Sens. Lett. 2015, 12, 2369-2373. [CrossRef]

20. Zhang, P.; Zhang, X.; Fang, G. Comparison of the Imaging Resolutions of Time Reversal and Back-Projection Algorithms in EM Inverse Scattering. IEEE Geosci. Remote. Sens. Lett. 2012, 10, 357-361. [CrossRef]

21. Cui, T.J.; Chew, W.C.; Yin, X.X.; Hong, W. Study of Resolution and Super Resolution in Electromagnetic Imaging for Half-Space Problems. IEEE Trans. Antennas Propag. 2004, 52, 1398-1411. [CrossRef]

22. Sekehravani, E.; Leone, G.; Pierri, R. PSF Analysis of the Inverse Source and Scattering Problems for Strip Geometries. Electronics 2021, 10, 754. [CrossRef]

23. Bertero, M.; Boccacci, P. Introduction to Inverse Problems in Imaging; IOP Publishing: Bristol, UK, 1998.

24. Devaney, A.J. Mathematical Foundations of Imaging, Tomography and Wavefield Inversion; Cambridge University Press (CUP): Cambridge, UK, 2012. 